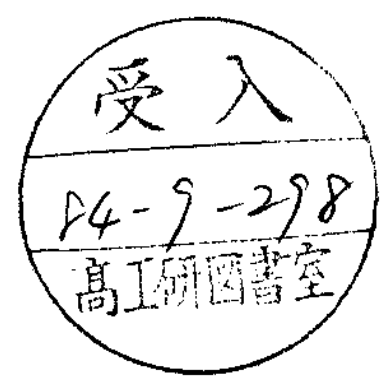

CERN-TH|. $3968 / 84$

\title{
IS SUPERSYMMETRY FOUND? *)
}

John Ellis

CERN -- Geneva

and

Marc Sher $* *$ )

University of California, Irvine

\section{ABSTRACT}

Monojet events seen recently by the UA1 collaboration at the CEKN p $\bar{p}$ Collider may be due to squarks or gluinos with masses $0(40)$ GeV. The thinness of the observed jets favours the squark interpretation. In this case, we predict that sleptons should have masses between 20 and $30 \mathrm{GeV}$ and that the photino should have a mass between 5 and 10 GeV. Such masses are close to the experimental lower limits and sparticles could soon be detectable in $\mathrm{e}^{+} \mathrm{e}^{-} \rightarrow(\tilde{\gamma} \tilde{\gamma}) \gamma$ experiments and $\mathrm{W}^{ \pm}$and $\mathrm{Z}^{0}$ decay. We demonstrate that such light sparticle masses are consistent with models whose weak gauge symmetry breaking is driven by $a$ t quark weighing $O(40) \mathrm{GeV}$ as recently reported, and even with no-scale models in which the supersymmetry breaking scale is also determined dynamically.

*) Supported in part by the director, office of Energy Research, office of High Energy and Nuclear Physics, Division of $\mathrm{High}$ Energy Physics of the U.S. Dept. of Energy under Contract $\neq \mathrm{DE}-\mathrm{AC} 03-76 \mathrm{SF} 0098$ and by N.S.F. Contract NSF-PHX-8305795.

**) Present1y at CERN, Geneva. Address af ter September 1, 1984: Univ. of California, Santa Cruz. 
The UAI collaboration has recently reported ${ }^{1)}$ the observation of monojet events with large missing $\mathrm{p}_{\mathrm{T}}$. The sma11 number of monojet events has been used to set lower limits of order of $40 \mathrm{GeV}$ on the gluino ${ }^{1)-3}$ ) and squark ${ }^{4), 5)}$ masses. The few observed monojets could be due to either squark $\tilde{\mathrm{q}} \rightarrow \mathrm{q}+$ photino $(\tilde{\gamma})$ decay, or to gluino $\tilde{g} \rightarrow q+\bar{q}+\tilde{\gamma}$ decay, if either $\underset{q}{q}$ or $\left.m_{g}=0(40) \mathrm{GeV}^{4}\right)$. The squark interpretation has been favoured ${ }^{4}$ ) on two grounds: (A) the hardness of the observed missing $\mathrm{p}_{\mathrm{T}}$ spectrum, which is more naturally explained by two-body $\tilde{\mathrm{q}} \rightarrow$ $q+\tilde{\gamma}$ decays, and (B) the thiness of the observed monojets, which disfavours $\tilde{g} \rightarrow$ $\mathrm{q}+\overline{\mathrm{q}}+\tilde{\gamma}$ decay which yields monojets with invariant masses up to $0(20) \mathrm{GeV}$ and an average of $0(10) \mathrm{GeV}$. Our first step in this paper is to firm up this latter argument by computing the perturbative gluon bremmstrahlung contribution to the squark decay jet width from $\tilde{q} \rightarrow(q+g) \tilde{y}$, finding that it gives $\left\langle m_{j e t}\right\rangle=0.05 \underset{q}{m}=$ $0(2) \mathrm{GeV}$ which is consistent with the observed monojets.

The main purpose of this paper is to develop the phenomenological consequences for other experiments if $\mathrm{m}_{\tilde{q}}=0(40) \mathrm{GeV}$ and $\underset{\mathrm{g}}{\sim} \underset{\mathrm{q}}{\mathrm{m}}$, and to explore consistency with theoretical models $\left.{ }^{6}-8\right)^{7}$. If the slepton and squark mass parameters are equal, at some grand unification renormalization scale of order of $10^{16} \mathrm{GeV}^{9}$ ), the the physical slepton, photino and gluino masses are calculable in terms of a few parameters which are tightly constrained by experiment. We find

$$
\begin{aligned}
20 \mathrm{GeV} & \leqslant m_{\tilde{l}} \leqslant 28 \mathrm{GeV} \\
6 \mathrm{GeV} & \leqslant m_{\tilde{\gamma}} \leqslant 8.5 \mathrm{GeV} \\
406 \mathrm{GV} & \leqslant m_{\tilde{g}} \leqslant 566 \mathrm{eV}
\end{aligned}
$$

if $\underset{\mathrm{q}}{\mathrm{q}} \approx 40-50 \mathrm{GeV}$. These predictions ${ }^{*}$ ) put the following sparticle production processes at the fxinge of observability in present experiments:

$$
\begin{aligned}
& e^{+} e^{-} \rightarrow(\tilde{\gamma} \tilde{\gamma}) \gamma, e^{+} e^{-} \rightarrow \tilde{e}_{R}^{+} \tilde{e}_{R}^{-}, p \tilde{p} \rightarrow \tilde{g} \tilde{g}+X \\
& p \bar{p} \rightarrow\left(W^{ \pm} \rightarrow \tilde{e}^{ \pm} \tilde{\nu}\right)+X, p \tilde{p} \rightarrow\left(z^{-} \rightarrow \tilde{l}^{+} \tilde{l}^{-} \text {or } \tilde{\nu}_{\nu}\right)+X
\end{aligned}
$$

ॠ)

It can be seen from Figs. 4 and 5 of Ref. 10) that such massive photinos do not contribute significantly to the present mass density of the Universe. 
There is a speculative class ${ }^{6), 7)}$ of supergravity models in which weak gauge symmetry breaking is dxiven by radiative corrections due to a "heavy" $t$ quark $\left(m_{t} \gg m_{b}\right)$ and $m_{W}$ is determined dynamically by dimensional transmutation: $\mathrm{m}_{W} \gg \mathrm{m}_{\bar{l}}$. We will show that the recent observation ${ }^{11)}$ of the $t$ quark with $m_{t}=$ $0(40) \mathrm{GeV}$ is consistent with these models if $\underset{q}{\mathrm{qr}} \approx 40-50 \mathrm{GeV}$. In this case, the ranges [Eq. (1)] of sparticle masses are further restricted, the stop mass is possibly lighter than the top quark ${ }^{12}$ ) and the lightest Higgs boson has a mass of a few $\mathrm{GeV}$. In these models, our present vacuum is unstable, but its lifetime before decay to the true vacuum is much longer than the present age of the Universe, for most of the allowed region of parameter space. There is an even more speculative class of models ${ }^{8}$ ) in which the supersymmetry breaking scale is also determined dynamically, which are also consistent with $\underset{q}{q}, m_{t}=0(40) \mathrm{GeV}$ and further constrain the sparticle masses.

As a preliminary to our subsequent discussion, we first discuss the size of jets in $\tilde{q} \rightarrow q+\tilde{\gamma}$ decay. The decaying $\tilde{q}$ is actually embedded in a colour singlet

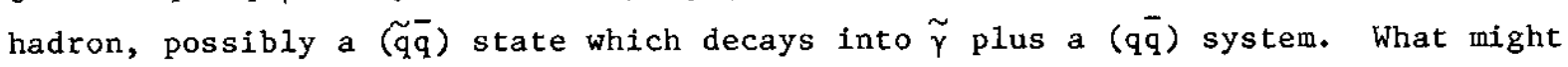
the invariant mass of this $(q \bar{q})$ system be ? One possible non-perturbative estimate would be to assign the spectator $\bar{q}$ a small non-relativistic momentum and then calculate the invariant mass when it is combined with the decay quark of mass zero and $E=p=\underset{q}{m_{q}} / 2 \approx 20 \mathrm{GeV}$ : such an estimate gives $\mathrm{m}_{\mathrm{q}} \overline{\mathrm{q}}=0(3) \mathrm{GeV}$. Alternatively, one can calculate the first-order perturbative QCD contribution to the jet invariant mass from gluon emission: $\tilde{q} \rightarrow(q+g)+\tilde{y}$. We find

$$
\frac{1}{\Gamma} \frac{d \Gamma}{d y}=\frac{\alpha_{s}}{6 \pi}\left(6+y-4 \ln y-\frac{z}{y}-\frac{4 \ln y}{y}\right)
$$

where $\mathrm{y} \equiv \mathrm{m}_{\mathrm{qg}}^{2} / \mathrm{m}_{\mathrm{q}}^{2}$. Calculating moments of this distribution using $\alpha_{s}=0.15$, we find

$$
\left\langle m_{q g}\right\rangle=0.05 m_{\tilde{q}} \approx 2 \mathrm{GeV}
$$

These estimates of the jet invariant mass are qualitatively consistent with the low invariant masses of the observed ${ }^{1}$ ) monojets ( $5 \mathrm{GeV}$ for event A including 
neutrals, $0.79 \mathrm{GeV}$ and $3.14 \mathrm{GeV}$ respectively for the charged particles in events $B$ and $D$, events $C$ and $E$ contain unreconstructed tracks). One can also try to use the perturbative calculation in Eq. (2) to estimate the charged multiplicity in $\mathrm{q}$ decay. Estimating $\mathrm{n}_{\mathrm{ch}} \approx 2\left[\mathrm{E}_{\mathrm{cm}}(\mathrm{GeV})\right]^{\frac{1}{2}}$ from $\mathrm{e}^{+} \mathrm{e}^{-}$annihilation data one has

$$
\left\langle n_{c h}^{\ddot{q}}\right\rangle \approx 2\left\langle m_{99}^{1 / 2}\right\rangle \approx 0.7 m_{\tilde{9}}^{1 / 2} \approx 4+05
$$

The observed events ${ }^{1)}$ are qualitatively consistent with this estimate if one allows for unreconstructed tracks in events $C$ and $E$. The " $\gamma$ " event $F$ may possibly be a monojet with a large collimated electromagnetic component containing one or more $\pi^{0}$ 's or $\eta^{\prime} s$, and whose charged multiplicity fluctuated down to zero. This event actually contains some soft charged tracks which are nearby in angle and could perhaps be assoclated in the "monojet". They exemplify the possibility that the quoted multiplicities could be increased by including soft tracks. We conclude that the observed monojets are qualitatively consistent with expectations $(2,3,4)$ for $\tilde{q} \rightarrow q \tilde{q}$ decay, whereas $\tilde{g} \rightarrow q+\bar{q}+\tilde{\gamma}$ decay for $\mathbb{m}_{g} \simeq$ $40 \mathrm{GeV}$ gives a bimodal distribution in $\mathrm{m}_{\text {jet }}$ peaked close to zero (only one $q$ or $\bar{q}$ in the monojet) and at $0(20) \mathrm{GeV}$ (both $q$ and $\bar{q}$ in the monojet), with a mean 0 (10) $\mathrm{GeV}$ which is experimentally disfavoured ${ }^{4}$. We therefore pursue the interpretation of the UAl monojet data as squarks with $\mathrm{m}_{\mathrm{q}} \approx 40 \mathrm{GeV}$ and infer a lower 1 imit $\operatorname{mon}_{\mathrm{g}} \geq 40 \mathrm{GeV}$ on the gluino mass.

Now we turn to predictions for other sparticle masses. In many supergravity models, the soft supersymmetry breaking mass terms for the squarks, sleptons and Higgses are universal when they are specified at some large renormalization scale $\mu>\mathrm{m}_{\mathrm{X}} \approx 10^{16} \mathrm{GeV}: \mathbf{m}_{\tilde{\ell}} \approx \underset{\mathrm{q}}{\mathrm{m}^{*}}$ ). Likewise, the $\operatorname{SU}(3), \operatorname{SU}(2)$ and $U(1)$ gaugino masses are equal at large scales: $m_{g}=m_{\tilde{W}}=m_{\tilde{B}}$. Just like the coupling constants and fermion masses in conventional GUTs, these mass parameters are renormalized by different amounts at low scales $\mu<\mathrm{m}_{\mathrm{X}}$, in a manner calculable using the renormalization group ${ }^{9}$. Convenient approximate formulae for the physical squark and slepton mass parameters at renormalization scales $\mu=$ $0\left(\mathrm{~m}_{\mathrm{W}}\right)$ have been produced by Kounnas et a1. ${ }^{7}$ :

$\bar{*}$

Our results are not greatly affected if one modifies this assumption in a plausible way. For example, in an SU(5) model, giving the $\overline{5}$ and 10 different masses will not significantly affect our bounds. 


$$
\begin{aligned}
& m_{\tilde{l}_{R}}^{2}=m_{0}^{2}+C_{E} m_{1 / 2}^{2} \quad\left(C_{E}=.149\right) \\
& m_{\tilde{l}_{2}}^{2}=m_{0}^{2}+c_{2} m_{1 / 2}^{2} \quad\left(c_{L}=.439\right) \\
& m_{\tilde{q}_{L}}^{2}=m_{0}^{2}+c_{Q} m_{1 / 2}^{2} \quad\left(c_{Q}=7.63\right) \\
& m_{\tilde{u}_{R}}^{2}=m_{0}^{2}+c_{v}^{m_{1 / 2}^{2}} \quad\left(C_{v}=7.29\right) \\
& m_{\tilde{d}_{R}}^{2}=m_{0}^{2}+c_{0} m_{1 / 2}^{2} \quad\left(c_{0}=7.24\right)
\end{aligned}
$$

where $m_{0}$ and $m_{\frac{1}{2}}$ are respectively the spin-zero and gaugino masses at $\mu=m_{X}\left(m_{0}\right.$ is commonly identified with the gravitino mass $m_{3 / 2}$, but may be different), the quoted squark masses refer to the five lightest quark flavours $u, d, s, c$ and $b$, and the numerical values quoted in parentheses on the right side of Eq. (5) apply to the minimal supersymmetric standard model with two Higgs doublets and with $\alpha_{s}\left(M_{W}\right)=0.122$, corresponding to the one-loop value of $\alpha_{s}$ for $\Lambda=100 \mathrm{MeV}$. We see from Eqs. ( $5 c)-(5 e)$ that the first five flavours of $q$ have approximately similar masses; evaluating the $C_{Q, U, D}$ at $\mu=M_{W} / 2$, we get

$$
m_{\tilde{q}}^{2} \approx m_{0}^{2}\left(1+7.6 \xi^{2}\right)
$$

where $\xi=\mathrm{m}_{\frac{1}{2}} / \mathrm{m}_{0}$. To determine the bounds on other sparticle masses, consider the case in which $\mathrm{m}_{\tilde{q}}=40 \mathrm{GeV}$. Requiring $\mathrm{m}_{\breve{\mathrm{e}}_{\mathrm{L}, \mathrm{R}}} \gtrsim 20 \mathrm{GeV}$, we see that $\xi^{2}$ is bounded above:

$$
\xi^{2}<.42
$$

giving uppex bounds on the photino and gluino ${ }^{*}$ masses:

*) We thank L. Hall and J. Polchinski for pointing out to us that the supersymmetric parameters are even more tightly constrained than we had originally thought. 


$$
\begin{aligned}
& m_{\tilde{\gamma}}=\frac{8}{3} \sin ^{2} \theta_{w} \frac{\alpha_{2}}{\alpha_{G U T}} m_{1 / 2}=.46 \xi m_{0} \leq 6.3 \mathrm{GeV} \\
& m_{\tilde{g}}=\frac{\alpha_{3}}{\alpha_{G U T}} m_{1 / 2} \approx 3.0 \xi m_{0} \leqslant 42 \mathrm{GeV}
\end{aligned}
$$

Thus, if $\underset{\mathrm{q}}{\mathrm{m}}=40 \mathrm{GeV}$, the gluino cannot be much heavier. If $\underset{\mathrm{q}}{\mathrm{m}}=50 \mathrm{GeV}$, however, it is possible for the gluino to weigh up to $56 \mathrm{GeV}$. Since all masses are calculated at the one-loop level, there is $0\left(\alpha_{s} / \pi\right)=0(5-10 \%)$ slop in all these mass estimates. However, taken at face value, they would suggest that $\mathrm{m}_{\mathrm{g}}$ is close to $\underset{q}{\mathrm{q}}$ and hence $\widetilde{q g}$ and $\widetilde{g g}$ production should also soon be detected at the CERN $\mathrm{sppS}$ collider. The slepton masses are also very tightly constrained:

if $\mathrm{m}_{\mathrm{g}} \approx \mathrm{m}_{\mathrm{q}}=40 \mathrm{GeV}$ :

$$
m_{\tilde{e}_{R}}=20 \mathrm{GeV}, \quad m_{\tilde{e}_{L}}=22 \mathrm{GeV}
$$

whtle if $\underset{\mathrm{g}}{\mathrm{m}}>\underset{\mathrm{q}}{\mathrm{m}}=50 \mathrm{GeV}$ :

$$
m_{\tilde{E}_{R}} \leq 25 \mathrm{GeV}, \quad m_{\tilde{e}_{2}} \leq 28 \mathrm{GeV}
$$

The upper limft (8b) on the gluino mass means that $\tilde{g g}$ pair production ${ }^{2), 4)}$ [and presumably $\widetilde{q g}$ associated production $\left.{ }^{13)}\right]$ should be accessible to the next round of

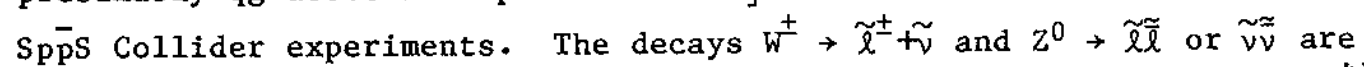
kinematically accessible and relatively copious: $\left.\Delta \mathrm{r}_{\mathrm{W}, \mathrm{Z}}=0(10 \%) \mathrm{r}_{\mathrm{W}, \mathrm{Z}}{ }^{*}\right)$. Phase space suppresses $W^{+}$and $Z^{0} \rightarrow \widetilde{q} \tilde{q}$ : the latter may only be observable at $\mathrm{e}^{+} \mathrm{e}^{-}$ machines such as SLC or LEP. The decay ${ }^{15)} \mathrm{W}^{+} \rightarrow \widetilde{W}^{+}+\tilde{\gamma}$ is also strongly suppressed in the class of supergravity models ${ }^{6)-8}$ discussed subsequently. The mass ranges (10) put sleptons within reach of $\mathrm{e}^{+} \mathrm{e}^{-}$experiments at TRISTAN and perhaps PETRA. Combining the limits (8a) on $\mathrm{m}_{\gamma}$ and $(10)$ on $\mathrm{m}_{\mathrm{e}_{\mathrm{R}, L}}$, we deduce that tha radiative annihilation reaction ${ }^{16)} \mathrm{e}^{+} \mathrm{e}^{-} \rightarrow(\widetilde{\gamma}) \gamma$ mediated by selectron exchange should have a cross-section close to the present experimental upper limit ${ }^{17)}$. This is shown in Fig. 1, together with the ranges of $m_{\tilde{\gamma}}$ and $\left\langle\mathrm{m}_{\tilde{e}}\right\rangle \equiv\left(\mathrm{m}_{\mathrm{e}_{\mathrm{R}}}^{-4}+\mathrm{m}^{-4} \mathrm{e}_{\mathrm{L}}\right)^{-1 / 4}$, which is the combination of mass parameters controlling the annihilation rate ${ }^{16)}$ for large $\mathrm{ma}_{\mathrm{e}}$. Plotted in Fig. 1 are theoretical predictions for $\underset{\mathrm{q}}{\mathrm{m}}=40,50 \mathrm{GeV}$ and $\mathrm{m}_{\mathrm{g}} \underset{\sim}{\sim}$ $40,50 \mathrm{GeV}$ : it is encouraging that the bulk of the allowed fan-shaped region is close to the present experimental upper limit on $\mathrm{e}^{+} \mathrm{e}^{-} \rightarrow(\tilde{\gamma \gamma}) \gamma$. The *) It may be possible 14 ) to detect these decays with a sample of $0(200-300) \mathrm{W} \rightarrow$
etv decays, as should soon be available from the Spp Collider. 
figure illustrates graphically how tightly constrained supersymetric models are if $\underset{\mathrm{q}}{\mathrm{q}} \approx 40 \mathrm{GeV}$. We have assumed in Fig. 1 that $\alpha_{\mathrm{s}}$ is given by its one-loop value for $\Lambda=100 \mathrm{MeV}$. For other values of $\alpha_{s}$, the shape of the fan-shaped region is completely unchanged, but the value of the photino mass changes proportionally to $\alpha_{s}^{-1}$. Thus, if $\Lambda$ were a bit smaller, say $50 \mathrm{MeV}$, which may be phenomenologically acceptable, then the largest allowed photino mass for $\underset{\mathrm{q}}{\mathrm{m}}=$ $40 \mathrm{GeV}$ changes from 6.3 to $7.0 \mathrm{GeV}$. This illustrates the above-mentioned slop of $5-10 \%$ in our masses.

So far we have neglected SU(2) breaking contributions to sparticle masses which axise when the two Higgs vacuum expectation values are different. These take the generic form ${ }^{9}$ )

$$
\delta m_{f}^{2}=m_{w}^{2}\left(T_{3}+\frac{Y}{2} \tan ^{2} \theta_{w}\right) \cos 2 \theta ; \cot \theta \equiv V_{1} / v_{2}
$$

where $\left.\mathrm{v}_{1} \equiv\left\langle 0\left|\mathrm{H}_{i}\right| 0\right\rangle\right\rangle(i=1,2)$ and $\mathrm{H}_{1}\left(\mathrm{H}_{2}\right)$ has $\mathrm{Y}=+\frac{1}{2}\left(-\frac{1}{2}\right)$. The contributions (11) vanish if $v_{1}=v_{2}\left(\theta=45^{\circ}\right)$, and we find that $\cos 2 \theta$ is constrained to be small if $\mathrm{m}_{\tilde{q}} \approx 40-50 \mathrm{GeV}, \mathrm{m}_{\tilde{\mathrm{g}}} \geqslant \mathrm{m}_{\tilde{\mathrm{q}}}, \mathrm{m}_{\mathrm{e}_{\mathrm{L}}, \mathrm{R}} \geqslant 20 \mathrm{GeV}$ and $\mathrm{m}_{\nu}^{2} \gtrsim 0$ so as to avoid lepton number violation generated by $\langle 0|\tilde{\nu}| 0\rangle \neq 0$. These constraints on $\xi$ and on $\cos 2 \theta$ are shown in Fig. 2. We find that if $\underset{q}{q}=40 \mathrm{GeV}$

$$
0.07 \geq \cos 2 \theta \geq-.12 \Rightarrow 43^{\circ}<\theta<48^{\circ}
$$

while $\xi$ can be at most $1 \%$ larger than was given by the previous upper bound (7). In view of the restrictions (12) on $\theta$, and because the specific supergravity models ${ }^{6)-8}$ ) with which we subsequently compare predict $\theta \approx 45^{\circ}$, we stick to this value in the rest of this paper.

So far, our analysis has not involved more theoretical assumptions than the equality of the squark and slepton masses at $\mu=0\left(m_{X}\right)$. Now we explore the further constraints that are imposed by the additional dynamical assumption ${ }^{6), 7}$ ) that weak gauge symmetry breaking is driven by a "heavy" $t$ quark $\left(m_{t} \gg m_{b}\right.$ ) which determines $m_{W}$ by dimensional transmutation: $m_{W} \gg m_{0}$ (in our case, $m_{W} / m_{0}=3$ to 4). Such models contain an additional supersymmetry breaking parameter $\left.{ }^{18}\right)_{A}$ which gives trilinear scalar interactions $A m_{3 / 2} P(\tilde{q}, \tilde{l}, H)$ where $P$ is the conventional superpotential. One expects $A=0(1)$, with $A=3$ befing the favoured value $^{19), 8)}$. We adopt the range of $m_{t}$ recently reported ${ }^{11)}$ by the UAl collaboration: $30 \mathrm{GeV}<\mathrm{m}_{t} \leqslant 50 \mathrm{GeV}$, and plot in Fig. 3 the allowed values of $A$ 
and $\xi$ for $\mathbb{\pi r}_{\mathrm{q}}=45,50 \mathrm{GeV}$. There is no interesting allowed domain for $\operatorname{ma}_{\mathrm{q}} \lesssim$ $40 \mathrm{GeV}$. We do not believe that this excludes consistency with $\mathrm{m}_{t}=40 \mathrm{GeV}$ and $\mathrm{m}_{\mathrm{q}}$ $=40 \mathrm{GeV}$, since as discussed above, all our calculations are at the one-loop leve1, and we expect two-loop corrections to be largest for $\mathrm{m}_{\mathrm{t}}$ and $\underset{\mathrm{q}}{\mathrm{q}}$, and generically $0\left(\alpha_{s} / \pi\right)=0(10 \%)$. If we take the one-loop calculations seriously, dimensional transmutation models favour the shaded domain of Fig. 1 in which

$$
\begin{aligned}
& 7 \mathrm{GeV}<m_{\tilde{\gamma}}<8.5 \mathrm{GeV} \\
& 47 \mathrm{GeV}<m_{\ddot{g}}<56 \mathrm{GeV} \\
& 17 \mathrm{GeV}<m_{\tilde{e}}<23 \mathrm{GeV}
\end{aligned}
$$

just outside the domain already excluded experimentally ${ }^{17}$ ). In the lower lefthand region of the allowed domains in Fig. 3, we find that the lighter stop squark has a mass in the range $\mathrm{m}_{t}>\mathrm{m}_{t}>\mathrm{m}_{t}-\mathrm{m}_{\gamma}$ so that it is 1ighter than the $t$ quark [and possibly as light as $0(20)^{l} \mathrm{GeV}$ if $\mathrm{m}_{t}=0(30) \mathrm{GeV}$ ] but the decay $t \rightarrow \tilde{\mathrm{t}}_{1}+\tilde{\gamma}$ (which would have overwhelmed the conventional $t \rightarrow b+\ell+\nu$ decay apparently seen 11 ) by UAl) is kinematically forbidden. It may be that TRISTAN (or even PETRA) can find the $\tilde{t}_{1}$ squark (which would decay into $b+\tilde{\gamma}^{+}(q \bar{q}$ or $l \bar{l})$ via $e^{+} e^{-} \rightarrow \tilde{t}_{1} \tilde{t}_{1}$, even if they cannot observe $\mathrm{e}^{+} \mathrm{e}^{-} \rightarrow t \bar{t}$. In this case ${ }^{12)}$, the decays of toponium $\theta$ would be very different from those conventionally expected: $\theta \rightarrow \tilde{t}_{1} \tilde{t}_{1}, \tilde{\gamma}_{\gamma}$ and perhaps $\tilde{g g}$. The mass of the lightest neutral Higgs boson is $\operatorname{smal1}^{7}$ ) in the allowed domain of Fig. 3, varying from 0 at the top left side to $0(3$ to 4$) \mathrm{GeV}$ in the bottom right corner: it should be produced in $\gamma$ and possibly $\mathrm{J} / \psi \rightarrow \mathrm{H}+\mathrm{Y}$ decays with a branching ratio which is twice the canonical rate for a single neutxal Higgs ${ }^{20}$ ). One final comment about the allowed domain in Fig. 3 is that our present vacuum is unstable throughout its extent, though this may not be a serious problem 6$), 21$ ). We have evaluated the bounce action for its transition to the true vacuum ${ }^{21}$ ), and find that it is greater than 400 (correspnding to a lifetime greater than $10^{10}$ years) in most of the allowed domain with the exception of a band near the top left side, with a typical value for $A=3.0$ and $\xi=0.75$ and $\mathrm{m}_{\mathrm{t}}=30 \mathrm{GeV}$ being $0(2000)$ corresponding to a lifetime of $0\left(10^{200}\right)$ years $^{*}$ ).

*) We found the true vacuum and evaluated the bounce action using the program of Claudson, Hall and Hinchliffe [Ref. 21)] which explores a larger parameter space than that discussed in Appendix $C$ of Kounnas et al. [Ref. 7)]. We thank M. Claudson for his generous and essential help in using this progran. 
Having found consistency with models ${ }^{6), 7}$ ) in which $m_{W}$ is determined dynamically by dimensional transmutation, we now examine a still more adventurous class of "no-scale" models ${ }^{8}$ ) in which $\mathrm{m}_{3 / 2}$ (and hence $\mathrm{m}_{0}$ ) is also determined dynamically. Such "nomscale" models have one less degree of freedom than the previous class, and correspond to the diagonal lines drawn across Fig. 3 for different values of $m_{t}$ and $\operatorname{man}_{q}$. Once again, since we only calculate at the oneloop level, we expect a "slop" in the lines of consistency corresponding to $\delta m_{t}$ or $\delta \mathrm{m}_{\mathrm{q}}=O(5-10) \mathrm{GeV}$. Therefore, we do not take detailed numerical values very seriously, but are encouraged by the qualitative overall consistency of these ideas with our interpretation of the UAl monojet data ${ }^{1}$ ).

If these events are indeed due to squark production, many current experiments are at the fringe of finding confirmatory evidence for supersymmetry. A partial list of places to look includes:

$$
\begin{aligned}
& e^{+} e^{-} \rightarrow(\tilde{\gamma} \tilde{\gamma}) \gamma \\
& e^{+} e^{-} \rightarrow \delta \tilde{e}_{\ell}, \tilde{t}_{1} \bar{t}_{1} \\
& p \bar{p} \rightarrow(\tilde{g} \tilde{q})+X \\
& p \bar{p} \rightarrow(\tilde{g} \tilde{g})+X \\
& p \bar{p} \rightarrow\left(W^{ \pm} \rightarrow \ell^{ \pm}+\tilde{\nu}\right)+X \\
& p \bar{p} \rightarrow\left(z^{0} \rightarrow \tilde{l} \tilde{l}_{0 .} \tilde{\nu} \bar{\nu}\right)+X \\
& r_{0 .} \mathrm{J} / \psi \rightarrow H^{\circ}+\gamma
\end{aligned}
$$

We may soon know the answer to the question raised in the title of this paper.

\section{ACKNOWLEDGEMENTS}

We thank D. Burke, R.N. Cahn, M.A. Claudson, L. Ha11, J. Polchinski, R. Prepost and B. Richter for useful discussions and help. We thank the Theoretical Physics Group at SLAC and LBL for their hospitality while we worked on this paper. 


\section{REFERENCES}

1) UAl Collaboration, G. Arnison et al,, Phys. Lett. 139B (1984) 115.

2) J. Ellis and H. Kowalski, CERN preprint TH.3843 (1984).

3) E. Reya and D.P. Roy, Dortmund Univ, preprints DO-TH 84/03,11 (1984).

4) J. Ellis and H. Kowalski, DESY preprint 84-045 (1984).

5) A.R. Allan, E.W.N. Glover and A.D. Martin, Durham Univ. preprint DTP/84/20 (1984);

V. Barger, K. Hagiwara and W.-Y. Keung, Univ. of Wisconsin preprint $\mathrm{MAD} / \mathrm{PH} / 183$ (1984).

6) J. Ellis, J.S. Hagelin, D.V. Nanopoulos and K. Tamvakis, Phys. Lett. 125B (1984) 275 .

7) C. Kounnas, A.B. Lahanas, D.V. Nanopoulos and M. Quiros, Phys. Lett. 132B (1983) 135 and Nuc1. Phys. B236 (1984) 438.

8) J. Ellis, A.B. Lahanas, D.V. Nanopoulos and K. Tamvakis, Phys. Lett. 1348 (1984) 429.

9) K. Inoue, A. Kakuto, H. Komatsu and S. Takeshita, Prog. Theor. Phys. 68 (1982) 927 and $71(1984) 453$.

10) J. Ellis, J.S. Hagelin, D.V. Nanopoulos, K.A. Olive and M. Srednicki, Nuc1. Phys. B238 (1984) 453 .

11) UA1 Collaboration, G. Arnison et al., (1984), as announced by M. Della Negra, seminar at CERN, and CERN press release PR 07.84 (1984).

12) J. Ellis and S. Rudaz, Phys. Lett. 128B (1983) 248.

13) H.E. Haber and G.L. Kane, UC Santa Cruz preprint UCSC-TH-169-84 (1984).

14) R.M. Barnett, H.E. Haber and K.S. Lackner, Phys. Rev. Lett. 51 (1983) 176 and Phys. Rev. D29 (1984) 1381.

15) S. Welnberg, Phys. Rev. Lett. 50 (1983) 387;

R. Arnowitt, A.H. Chamseddine and P. Nath, Phys. Rev. Lett. 50 (1980) 1232;

J. Ellis, J.S. Hagelin, D.V. Nanopoulos and M. Srednicki, Phys. Lett. 127B (1983) 233;

R.M. Barnett and H.E. Haber, Institute for Theor.'Phys., Santa Barbara preprint NSF-ITP-84-78 (1984).

16) P. Fayet, Phys. Lett. 117B (1982) 460;

J. Ellis and J.S. Hagelin, Phys. Lett. 122B (1983) 303;

K. Grassie and P.N. Pandita, Dortmund Univ. preprint DO-TH83/23 (1983);

T. Kobayashi and M. Kuroda, Phys. Lett. 139B (1984) 208;

J.D. Ware and M.E. Machacek, Northeastern Univ. preprint NUB-2626 (1984).

17) MAC Collaboration, presented at the Vanderbilt Conference, 1984, and R. Prepost, private communication (1984). 
18) B. Julia et al., Phys. Lett. 79B (1978) 23 and Nuc1. Phys. B147 (1979) 105; E. Cremmer, S. Ferrara et al. Phys. Lett. 116B (1982) 231 and Nucl Phys. B212 (1983) 413; H.-P. Nilles, M. Srednicki and D. Wyler, Phys. Lett. 120B (1982) 324.

19) E. Cremmer, P. Fayet and L. Girardello, Phys. Lett. 122B (1983) 41.

20) F.A. Wilczek, Phys. Lett. 39 (1977) 1304 .

21) M.A. Claudson, L.J. Hall and L. Hinchliffe, Nucl. Phys. B228 (1983) 501. 


\section{FIGURE CAPTIONS}

Fig. 1: Regions of $\frac{\mathrm{m}}{\mathrm{e}}$ and $\underset{\gamma}{\gamma}$ consistent with the MAC experiment ${ }^{17)}$ (indicated by arrows), and with $\underset{q}{m_{q}}=40,50 \mathrm{GeV}, \mathrm{m}_{\mathrm{g}} \gtrsim 40,50 \mathrm{GeV}$. The shaded domain is that allowed in dimensional transmutation models. Since $\mathbb{m}_{\tilde{e}_{\mathrm{L}}}$ and $\mathfrak{m}_{\mathfrak{e}_{\mathrm{R}}}$ are nearly equal, the selectron mass is $2^{1 / 4}$ m

Fig. 2: The domains of $\cos 2 \theta: \cos \theta \equiv \mathrm{v}_{1} / \mathrm{v}_{2}$ and $\mathrm{m}_{\mathrm{g}}$ which are consistent with $\mathrm{m}_{\tilde{\mathrm{q}}}=40 \mathrm{GeV}$ (solid lines) or $50 \mathrm{GeV}$ (dashed lines), $\mathrm{m}_{\mathrm{e}_{\mathrm{R}}} 220 \mathrm{GeV}, \mathrm{m}_{\tilde{\mathrm{g}}}>$ $\mathrm{m}_{\mathrm{q}}$ and $\langle 0|\tilde{\nu}| 0\rangle=0$.

Fig. 3: Domains of $A$ and $\xi \equiv \mathfrak{m}_{1} / \mathfrak{m}_{0}$ which are consistent with dimensiona1 transmutation models ${ }^{6}, \frac{1}{2} 7$ for different values of $m_{t}=30,40,50 \mathrm{GeV}$ and (a) $\underset{q}{m_{q}}=50 \mathrm{GeV}$, (b) $\underset{q}{a}=45 \mathrm{GeV}$. As can be seen in Fig. 1, at the one loop level there are no consistent dimensional transmutation models with $\mathrm{m}_{\mathrm{q}} \lesssim 43 \mathrm{GeV}$. The diagonal wavy lines correspond to the additional constraint provided by no-scale models ${ }^{8}$ ). Here $\Lambda$ was chosen to be 100 $\mathrm{MeV}$. If it is larger, the size of the allowed region in (a) shrinks (from right to left), disappearing for $\Lambda=300 \mathrm{MeV}$. We have also assumed the boundary condition $B=A-1$, which is not necessarily valid in models with non-minimal kinetic terms. Changing the value of $B$ does change the value of A somewhat, but the general shape and size of the figure does not change significantly, nor does the shaded region of Fig. 1 . 


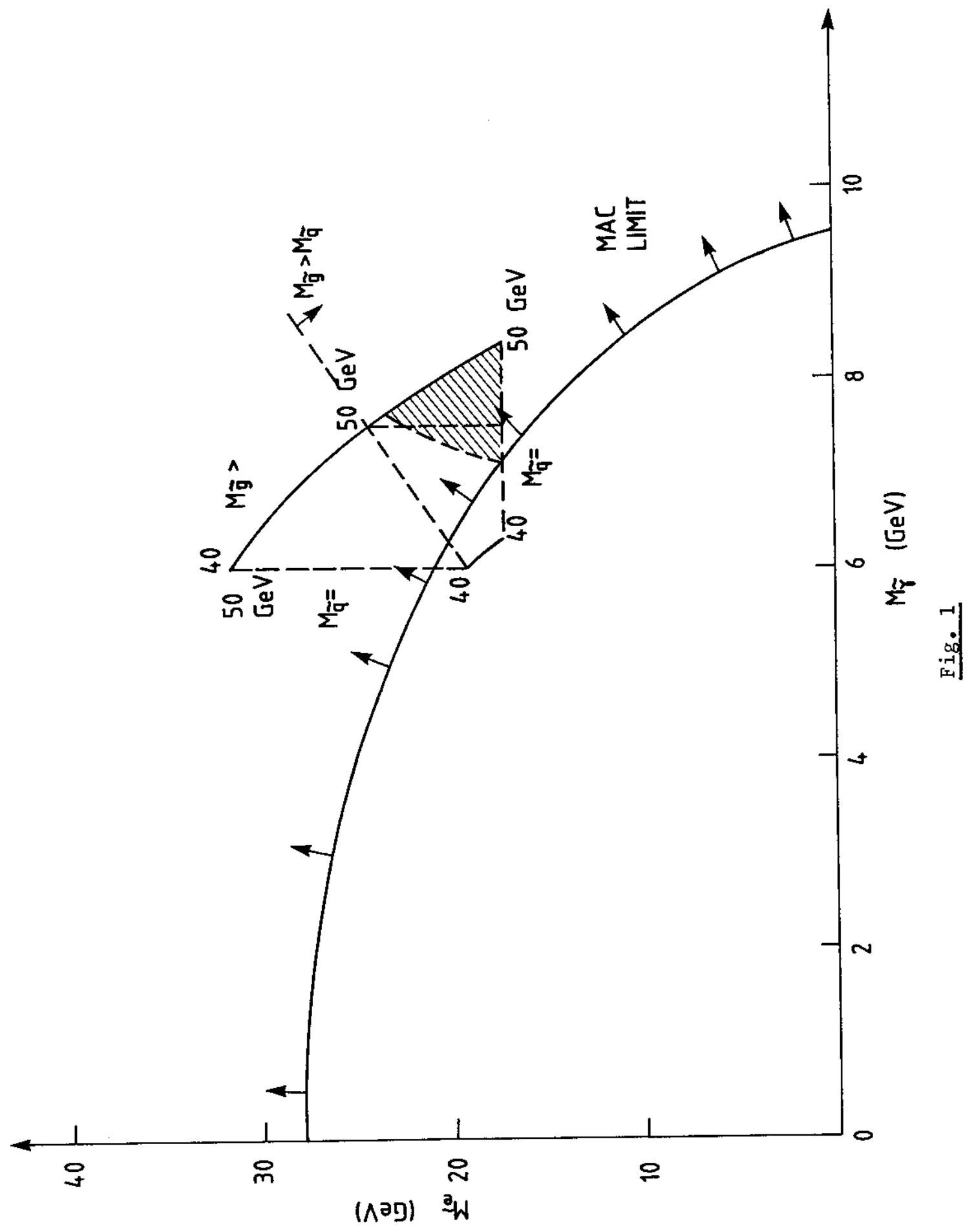




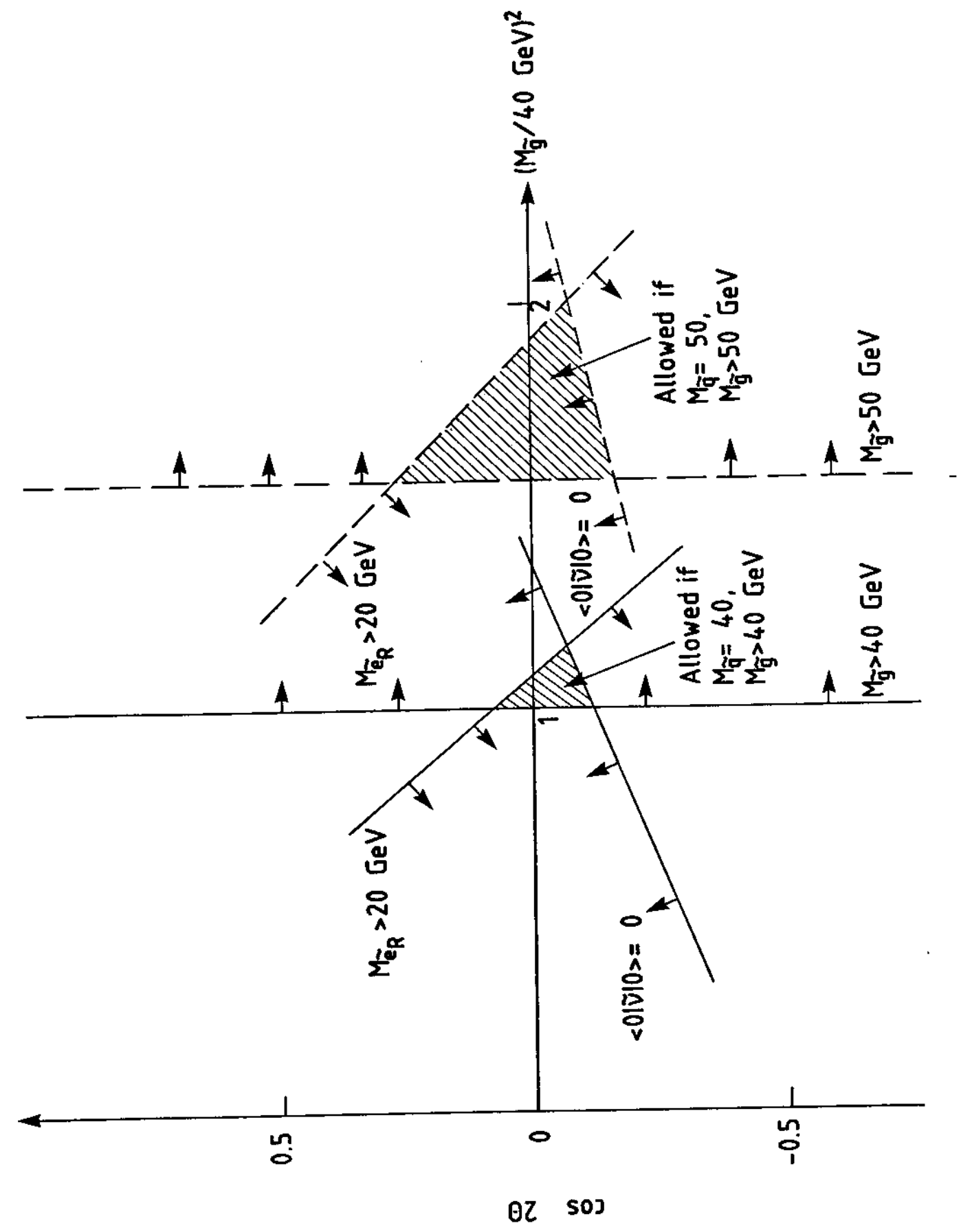

0
0
0
$0=1$ 

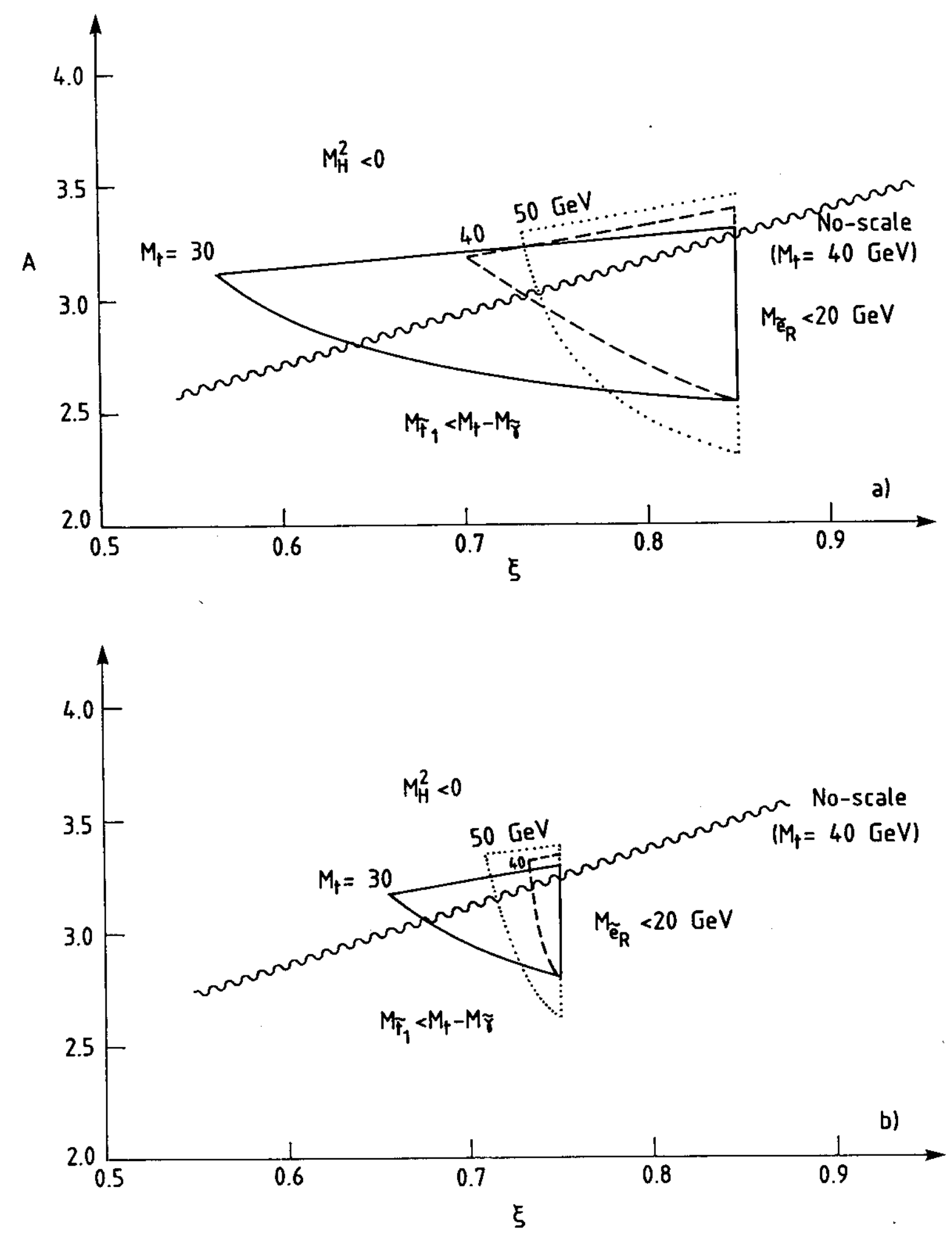

Fig. 3 